\title{
PAPÉIS DE AUTOCUIDADO - SUBSÍDIOS PARA ENFERMAGEM DIANTE DAS REAÇÕES EMOCIONAIS DOS PORTADORES DE DIABETES MEUTUS
}

\author{
Self-Care Roles - Subsidy to the Nursing before the \\ Emotional Reactions of the Diabetes Mellitus Carriers \\ Papeles de Autocuidado - Subsídios para Enfermería en \\ Ias Reacciones Emocionales de los Portadores de Diabetes Mellitus
}

Carlos Victor dos Santos Filho

Wilma Helena Carvalho Rodrigues²

Rita Batista Santos ${ }^{3}$

\begin{abstract}
Resumo
0 artigo foi elaborado a partir do estudo dos resultados de duas dissertações de mestrado (Estudo 1 e 2) e de uma tese de doutorado (Estudo 3). Foram identificadas reações emocionais comuns e freqüentes nos portadores de diabetes mellitus (DM), que comprometem o desempenho dos papéis de autocuidado. Teve como objetivos: apresentar as reações emocionais identificadas em portadores de DM tipo 1 e 2 e discutir as interferências das reações emocionais para desempenho dos papéis de autocuidado. Dos resultados, emergiram as categorias de reações emocionais: medo, raiva/ revolta, tristeza, depressão e estresse/ansiedade. Evidenciou-se ser a DM uma doença que, independente do tipo e da faixa etária, causa um impacto negativo biopsicossocial. Concluiu-se que há necessidade de uma abordagem mais ampla para a relação entre as reações emocionais e o desempenho das ações de autocuidado dos portadores de DM.
\end{abstract}

Palavras-chave: : Diabetes Mellitus. Autocuidado. Enfermagem.

\begin{abstract}
The article was elaborated based on the study of the two thesis master of degrees (Study 1 and 2) and of a doctorate thesis (Study 3). Had been identified reactions emotional common and frequency in the patients with Diabetes Mellitus (DM), being able to compromise the action performance of papers of self-care. We have as objective: to present reactions emotional identified in patients with DM they intervene with the action performance of papers of self-care and arguing the emotional the interferences of for the self-care. Of the results the emotional categories of reactions had emerged: fear; anger, bad behavior, depressive feelings and stress/ anxiety. Evidencing to be independent DM an illness of the type and the age band it is the cause of a biopsychosocial negative impact. It is concluded the necessity of a larger approaching related to the emotional reaction and the action performance of self-care roles of patients with DM.
\end{abstract}

\section{Resumen}

El artículo fue elaborado basó en el estudio del año de dos tesis de bachillerato (estudio 1 y 2) y de una tesis del doctorado (el estudio 3). Habían sido el campo común y la frecuencia emocionales identificados de las reacciones en los pacientes con la Diabetes Mellitus (DM), pudiendo comprometer el funcionamiento de la acción de papeles del auto-cuidado. Tenemos como objetivo: para presentar a reacciones emocional identificado en pacientes con DM que él interviene con el funcionamiento de la acción de papeles del autocuidado y de discutir las interferencias para el uno mismo-cuidado. De los resultados las categorías emocionales de reacciones habían emergido: miedo; cólera, mal comportamiento, depresiva stress/ ansiedad . El evidenciar a ser la independiente DM a la enfermedad del tipo y la edad causa un impacto negativo biopsicosocial. Se concluí la necesidad de un amplio estudio para la relación entre las reacciones emocionales y el funcionamiento de la acción de papeles del auto-cuidado en pacientes con el DM. 


\section{INTRODUÇÃO}

Existem reações emocionais comuns e freqüentes, não só diante do diagnóstico de diabetes mellitus (DM), como também no convívio e no manejo da doença, identificadas em portadores de DM dos tipos 1 e 2 .

As reações emocionais precisam ser mais bem compreendidas pelos enfermeiros, uma vez que muitas destas são manifestadas através de comportamentos inadequados que interferem no bom controle glicêmico, o que repercute na saúde, nas relações sociais e, por extensão, na qualidade de vida do portador.

Segundo a Sociedade Brasileira de Diabetes $(S B D)^{1}$, o DM é uma síndrome de etiologia múltipla, decorrente da falta de insulina e/ou da incapacidade da insulina em exercer adequadamente seus efeitos, constituindo uma condição crônica que exige do portador um contínuo autogerenciamento do estilo de vida e adaptação à doença.

A Enfermagem, como integrante da equipe multidisciplinar de saúde, tem o desafio de instrumentalizar o portador no autogerenciamento da doença, buscando estratégias e novas formas para um melhor cuidado de enfermagem, otimizando o controle glicêmico.

Para este estudo, adotamos Santos ${ }^{2}$ como referencial teórico, sendo os papéis de autocuidado decodificados nas principais ações que emergem do cuidado junto aos portadores de DM, para que, com autonomia, autogerenciem a doença com vistas ao bem-estar e à qualidade de vida.

Os papéis de autocuidado são: A) manutenção para a saúde; B) prevenção das complicações agudas e crônicas; C) autodiagnóstico, autotratamento e automedicação no DM; D) participação ativa nos serviços de saúde.

Esse entendimento traz à tona 0 portador como ator principal das ações e, desta forma, o torna autor responsável e autônomo do desempenho do seu autocuidado.

As interferências causadas pelas reações emocionais emergem da necessidade de cuidados incessantes e repetitivos para o controle do DM e como expressão da percepção individual emocional, causando maior dificuldade para o desempenho das ações contidas nos papéis de autocuidado e, conseqüentemente, para a adesão ao tratamento.

\section{OBJ ETIVOS}

Este ar tigo teve como objetivos:

- Apresentar as reações emocionais identificadas em portadores de DM tipos 1 e 2;

- Discutir as interferências das reações emocionais para desempenho dos papéis de autocuidado.

\section{REFERENCIAL TEÓRICO: OS PAPÉIS DE AUTOCUIDADO}

Os papéis de autocuidado foram adaptados e apresentados por Santos ${ }^{2}$ de Fry apud Hill $\&$ Smith ${ }^{3}$ para os portadores de DM e categorizados em:

A - Manutenção da saúde: manter a saúde significa sustentar uma vida com melhor qualidade através do desempenho de ações que convergem para 0 autocuidado, possibilitando efetivamente uma condição de saúde. Para manutenção da saúde, destaca-se: evitar o tabagismo; evitar o uso de bebidas; manter o peso corporal adequado; realizar uma alimentação balanceada; manter esquema de vacinação atualizado; praticar atividades religiosas; realizar atividades de lazer regularmente; combater o estresse com atividades alternativas e praticar atividade física regular.

B - Prevenção das complicações agudas e crônicas do DM: consiste na prevenção das complicações de forma a manter o equilibrio da doença. Neste momento as atividades educativas individuais e/ ou em grupo são instrumentos utilizados de maneira coordenada. A reeducação alimentar, a realização das glicemias capilares, a realização da hemoglobina glicosilada e da frutosamina periodicamente, assim como o desempenho de práticas específicas para 0 controle glicêmico adequado, minimizam ou impedem as complicações agudas ou crônicas decorrentes do mau controle glicêmico.

C - Autodiagnóstico, autotratamento e automedicação no DM: o reconhecimento dos sinais e sintomas da hiperglicemia e da hipoglicemia são pontos importantes para prevenção das complicações agudas e crônicas. Assim como o conhecimento do autoajuste da terapêutica, conforme automonitorização da glicemia capilar periférica.

D - Participação ativa em serviços de saúde: são todas as ações que incluem o uso das estruturas disponíveis de apoio em saúde, para que os portadores se tornem ativos nas decisões sobre seu tratamento, visando à continuidade da sua autonomia e independência.

\section{INTER-RELAÇÃO ENTRE AS REAÇÕES EMOCIONAIS E OS PAPÉIS DE AUTOCUIDADO}

Diante do diagnóstico da doença e da explicação do tratamento proposto, sentimentos de angústia e ansiedade são comuns tanto nos portadores do tipo 1 como nos do tipo 2. Isso é exacerbado em alguns casos do tipo 1 pelos pais e familiares que, ao tentarem superproteger as crianças, desvelam um sentimento de culpa pela doença dos filhos, conforme Graça e cols ${ }^{4}$.

Outra reação importante e multifatorial é a depressão, que, segundo Lutsmans ${ }^{5}$, é universal e onde fatores biológicos, genéticos e psicológicos estão envolvidos.

Alguns portadores do tipo 2 vêem a doença como um fardo difícil de carregar. Este pessimismo dificulta o desempenho dos papéis de autocuidado.

Gimenes $^{6}$ enfatiza a necessidade de estratégias de enfrentamento das condições de tolerância e adaptação de possíveis eventos negativos, sendo fundamental a construção de uma imagem positiva diante dos eventos, possibilitando um equilíbrio emocional, mantendo o bem-estar físico e mental.

Na relação enfermeiro e portador, os aspectos emocionais, psicossomáticos e o relacionamento com familiares devem ser considerados elementos para o bom controle da doença. 


\section{METODOLOGIA}

Trata-se de uma pesquisa descritiva, com abordagem qualitativa da análise dos resultados de estudos realizados a respeito do autocuidado de portadores de DM tipo 1 e 2 na cidade do Rio de Janeiro.

Flegner e Dias ${ }^{7}$ afirmam que os estudos descritivos se preocupam com a descrição de um fenômeno, delineando o que é e abordando quatro aspectos: descrição, registro, análise e interpretação de fenômenos atuais, objetivando o seu funcionamento no presente.

Para Lüdke e André ${ }^{8}$, a abordagem qualitativa oferece condições de pesquisar aqueles fatos e fenômenos, verificando como se manifestam nas atividades, nos procedimentos e nas interações cotidianas vivenciadas pelas pessoas.

Os dados foram levantados no capítulo de resultados dos estudos denominados 1, 2 e 3, onde se buscou identificar as reações emocionais que interferiam no desempenho dos papéis de autocuidado.

0 Estudo $1^{9}$ corresponde a um estudo descritivo qualitativo com oito escolares portadores de DM tipo 1, em um hospital especializado em Diabetes e Endocrinologia. 0 Estudo $2^{10}$ refere-se a um estudo descritivo com 11 portadores de DM tipo 2, na faixa etária de 50 a 76 anos, em um hospital geral terciário. 0 Estudo $3^{2}$ foi um estudo de caso qualitativo, realizado com sete servidores de uma universidade federal, com idade entre 44 a 66 anos, atendidos na consulta de enfermagem do Programa de Atenção ao Trabalhador com Diabetes Mellitus da divisão pericial desta instituição.

Uma vez identificadas as reações emocionais comuns nos estudos, utilizando como critério a semelhança dos resultados dos estudos, os dados foram agrupados, possibilitando a elaboração de categorias conforme os objetivos propostos e a construção de um quadro de distribuição desses dados segundo as categorias, e analisados à luz da literatura.

Cabe ressaltar a obediência aos aspectos estabelecidos das Diretrizes Éticas Internacionais para a pesquisa envolvendo seres humanos. Conforme Resolução 196/96 do Conselho Nacional de Saúde ${ }^{11}$, o projeto do Estudo 1 foi submetido ao Comitê de Ética e Pesquisa (CEP) do Instituto Estadual de Diabetes e Endocrinologia Luiz Capriglione - IEDE, sendo aprovado e obtendo registro número 195 através do parecer 79/2006; o Estudo 2 foi submetido ao Comitê de Ética e Pesquisa (CEP) da Escola de Enfermagem Anna Nery da Universidade Federal do Rio de Janeiro - UFRJ, obtendo registro número 046/2006; e, finalmente, o Estudo 3 foi submetido ao Comitê de Ética e Pesquisa (CEP) do Hospital São Paulo da Universidade Federal de São Paulo - UNIFESP, sendo aprovado pelo parecer 480/1999.

\section{APRESENTAÇÃO E DISCUSSÃO DOS RESULTADOS}

As reações emocionais identificadas a partir dos resultados dos estudos e elaboradas em categorias foram: medo, tristeza, depressão, raiva/revolta e ansiedade/ estresse, conforme apresentados na Tabela 1:
Tabela 1:

Distribuição das categorias de reações emocionais segundo os Estudos, Rio de Janeiro 2006.

\begin{tabular}{|c|c|c|c|}
\hline Reações emocionais & Estudo 1 & Estudo 2 & Estudo 3 \\
\hline MEDO & 1 & 2 & 4 \\
\hline TRISTEZA & 3 & 1 & 3 \\
\hline DEPRESSÃO & 1 & 3 & - \\
\hline RAIVA/REVOLTA & 3 & 3 & 1 \\
\hline ANSIEDADE/ESTRESSE & - & 3 & 5 \\
\hline
\end{tabular}

Na Tabela 1 foi apresentada a distribuição pelos estudos 1, 2 e 3 das categorias de reações emocionais identificadas a partir das falas dos participantes, sendo importante destacar que algumas diferenças encontradas são decorrentes das especificidades do cenário, dos sujeitos e da amostra dos estudos em questão.

Mediante os resultados apresentados, ficou evidenciado que a forma e a maneira com que os portadores reagem diante da doença interferem direta e indiretamente no desempenho dos papéis de autocuidado, cabendo ao enfermeiro o papel de mediador da relação reação emocional e o desempenho das ações de autocuidado.

As reações emocionais causam um impacto na categoria de Manutenção da Saúde, consideradas a base dos papéis de autocuidado, uma vez que expressam as crenças, valores, temores, anseios e receios em relação à doença que todo portador de DM e de outras condições crônicas pode enfrentar.

Os estudos apontaram reações comuns como: raiva, revolta e depressão, o que mostra ser o DM uma condição que, independente da sua etiologia e faixa etária, causa profundas alterações biopsicossociais.

Chiozza $^{12}$ analisa a questão da formação da identidade segundo a teoria psicanalítica, relacionando-a com o distúrbio do DM. Estabelece ainda a relação entre as doenças emergentes e o fato de o DM ocupar a $3^{\text {a }}$ causa de morte no mundo, baseado na crença de que em cada época predominam determinados transtornos somáticos. Explica também que existe um núcleo significativo para a formação comum entre o que ocorre na sociedade e no DM na atualidade.

Em se tratando dos portadores de DM1, principalmente crianças em idade escolar, essas reações comprometem as relações sociais, levando a um afastamento do grupo, principalmente no que se refere às atividades festivas, nas quais a restrição alimentar fica evidenciada. Cabe ao enfermeiro a busca de estratégias de reintegração social, fortalecendo os princípios de convivência com o grupo, sem que haja comprometimento do controle da glicemia.

0 DM tipo 1 pode ser um fator que delimita o desenvolvimento das relações sociais, convergindo para um estado de baixa autoestima, repercutindo em um fraco controle metabólico e acarretando problemas de adesão ao tratamento, surgimento de complicações agudas e crônicas e de dificuldades psicossociais.

As falas dos portadores de DM tipo 1 presentes no Estudo 1 revelaram que as reações emocionais emergem de forma natural e que estão vinculadas de maneira ambivalente a sentimentos entre fazer aquilo que deseja e o que deve ser feito. 
"Claro, a gente não pode comer doces nas festas, isto é ruim... na escola é pior, todo mundo comendo bala na frente... fico até com pena.... pena de mim mesmo... eu fico.. Eu fico com raiva, porque quando ela está alta, eu tenho que ficar dando injeção, dá uma raiva... Eu não... Eu aplicava, aí um dia doeu, nunca mais apliquei, fiquei com trauma...Pavor... (PETER PAN - 10 anos)

"Ah! Fico triste... Na escola os outros podem fazer um lanche, na rua também e fica pior quando os outros te oferecem.(MICKEY - 11 anos)

As limitações impostas pelo controle do DM, dentro de uma relação direta com as restrições da dieta, deixam transparente a dificuldade que o escolar portador de DM1 enfrenta na participação e integração das atividades festivas. Na maioria das vezes, as guloseimas fazem parte do dia-a-dia neste período da vida compreendido como idade escolar.

Ansiedade, Estresse, Revolta, Raiva, Medo, Tristeza e Depressão são alguns dos sentimentos identificados nas falas no Estudo 2, levantando o desafio de motivar um corpo a se autocuidar quando esse corpo que sofre não vê perspectivas reais e concretas de cura, ou de melhora.

“- Eu não sei qual é a relação entre diabetes e a ansiedade, esse meu estado emocional, esse negócio dessa perna, está me deixando meio depressivo... A minha vontade é tomar chumbinho em vez de tomar insulina, a gente faz tudo, faz tudo e de repente não deu resultado. (PRETO, Homem, 68 anos).

“- Eu dou graças a Deus por estar vendo, tenho muito medo da amputação. Também acho que nós diabéticos devemos ter um cuidado ferrenho, mesmo porque a gente está predisposta a essas amputações, à cegueira... Então a gente é obrigada a se cuidar mesmo..., Se tem um problema no dedo a gente que trabalha fora..." (BRANCA, Mulher, 55 anos).

A forma com que estes clientes entendem ou enfrentam a doença esta diretamente relacionada ao autocuidado que irá desempenhar, cabendo ao enfermeiro o papel de mediador da relação reação emocional e autocuidado.

0 estresse esteve presente à atividade laborativa de cinco participantes do Estudo 3, seja por problemas relacionados à tarefa, ou por fatores ligados a sua vida em sociedade, em família. Os fatores que precipitam o estresse foram tão variados quanto os sujeitos que o vivenciaram. 0 DM, por si só, pode representar um agente estressor.

"Olha eu entendo uma coisa, se a pessoa não controlar, ele não vai agüentar trabalhar, porque quando você tá com açúcar alto dá tontura, você fica mole, sem disposição. Como é que você vai fazer pra trabalhar? Então tem que se controlar, tem que fazer tudo pra controlar o açúcar, a glicose, e baixar. Até ele (o médico) me mandou uma carta aíporque quando eu pego... se pegar um formato de papel grande, que às vezes tem papel grande, pesado, aí eu tenho que pegar os outros dedos, senão cansa, eu fico sem destreza, né. Então se for pesado eu digo "não dá". Aí tem que botar um homem pra trabalhar comigo, (Jasmin)
"E sobre a comida eu tô... de manhã eu não como nada, mas quando dá $8 h, 8$ e pouco, meu estômago já está doendo. ... Eu falei cá ... a Dra. lá. ... Não tô conseguindo manter minha dieta. ... Eu como acima do limite que ela mandou. ... Eu como bem. ... Duas bananas, biscoito, adoçante, pão com manteiga, café com leite. ... Só à noite ... comida normal. ... Normal, suco..." (Gardênia)

Chiozza $^{12}$ analisa a polifagia como um estado em que 0 instinto de comer já não compensa o conflito neurótico subjacente, podendo ser localizado na classificação de distúrbios paranóicos, agressivos e depressivos.

0 medo, a angústia e a insatisfação no trabalho podem gerar formas clínicas de sofrimento que no sujeito portador de DM podem resultar em hipoglicemia ou hiperglicemia a depender de suas características e hábitos de saúde.

A expressão de sentimentos de desconfiança, insatisfação e indignação no processo da doença interferem no autocuidado, podendo ser identificados nos portadores como manifestação de raiva e revolta.

Segundo Chiozza ${ }^{12}$, os portadores de DM possuem algo somático e psicológico em comum, independente da singularidade própria de cada um deles. Esse componente é a fantasia insulino-pancreática inconsciente, cujo significado corresponde especificamente ao distúrbio do DM. Tais fantasias são representações corporais inconscientes, que emanam das qualidades específicas dos órgãos que as originam. Acredita que, em sentido figurado, a ação da insulina ativa a glicose do mesmo modo que um fósforo acende um carvão, ou um combustível é utilizado para o gasto energético implícito no fazer, empreender ou construir algo.

Segundo Dakle ${ }^{13}$, a visão da melancolia como um desequilíbrio interno localiza-a no modelo endógeno de explicação e tratamento das doenças apontado por Laplantine ${ }^{14}$, podendo indicar a abordagem psicológica psicanalítica e psicossomática do DM, já que o DM se configura num conflito essencialmente intrapsíquico, em que se devem trabalhar as fantasias e as modificações das reações do homem com seu meio, entre as próprias instâncias do ego e do superego. Acredita que a doença física é a expressão psicológica, simbolização dos desejos secretos dos sujeitos, que devem ser desvendados.

As experiências de vida e trabalho refletiram no cotidiano do autocuidado e do estado geral de saúde, acarretando-lhes problemas físicos e psíquicos.

\section{CONSIDERAÇÕES FINAIS}

0 DM revelou ser uma doença que, independente da faixa etária e da etiologia, causa um impacto negativo na vida biopsicosocial, necessitando um urgente olhar cada vez mais amplo por parte do enfermeiro para a relação entre as reações emocionais e as ações dos papéis de autocuidado dos portadores de DM.

Tais reações acarretam: a depreciação da autoimagem; o isolamento social; a desestruturação da célula familiar e, conseqüentemente, o não-cumprimento das ações de autocuidado, exigindo do enfermeiro a busca de estratégias de intervenções para facilitar e promover as ações dos papéis de autocuidado. 
Da mesma forma, os resultados também evidenciaram semelhanças na prevalência das reações emocionais manifestadas entre os portadores de DM1 e DM2. Ratifica-se a importância das estratégias adaptadas à faixa etária para superar as interferências dos aspectos emocionais no desempenho dos papéis de autocuidado. Tem-se nas atividades de grupo um instrumento valioso para facilitar a adesão e a motivação do desempenho das ações de autocuidado, minimizando a ocorrência das complicações agudas e crônicas.

É possível e necessária a construção, junto ao portador, de uma visão não só do DM, mas sim da sua própria existência, permeada pelas reações emocionais com vistas à qualidade de vida. Como mensagens importantes para compreensão das interferências das reações emocionais para o autocuidado, têm-se:

- que a sensibilização do portador para o desempenho dos papéis de autocuidado nasce e tem a sua base no próprio portador;

\section{Referências}

1. Sociedade Brasileira de Diabetes. Consenso brasileiro sobre diabetes. Arq Bras Endocrinol Metabol 2000 set; 44: 4 (supl 1): 58.

2. Santos RB. Autocuidado, trabalho e diabetes mellitus: uma abordagem de enfermagem. 2001. [tese de doutorado] São Paulo (SP): Escola Paulista de Medicina/ UNIFESP; 2001.

3. Hill LSN. Self care nursing: promotion of health. New Jersey (USA); 1990. p .447.

4. Graça LAC, Burd MMJ. Grupos de diabéticos. Grupo e corpo: psicoterapia de grupo com pacientes somáticos. Porto Alegre (RS): Artes Médicas; 2000. p.213-32.

5. Lutsaman PJ, Anderson RJ, Freediand KE, Groot M, Carney RM, Clouse RE, Depression and poor glycemic control: a meta-analytic review of the literature. Diabetes Care 2000 jun; 23(7): 934

6. Gimenes MG. A mulher e o câncer. Campinas (SP): DP Psy ; 1997.

7. Flegner AJ, Dias JC. Metodologia da pesquisa científica. Rio de Janeiro (RJ); 1994.

8. Ludke M, André MFDA. Pesquisa em educação: abordagem qualitativa. São Paulo (SP): EPU; 1986. -que o autocuidado deve ser feito dentro de uma perspectiva da integralidade de um corpo segundo o seu contexto histórico social:

-que a participação dos serviços de saúde, dos familiares e de grupos deve ter um caráter de condução e acompanhamento desse portador no desempenho dos papéis de autocuidado;

- que o enfermeiro tem nas ações educativas a sua principal ferramenta no processo de desenvolvimento dos papéis de autocuidado.

A promoção do autocuidado é uma abordagem simples e factível de alcance dos níveis glicêmicos preconizados e, conseqüentemente, da diminuição das complicações agudas e crônicas, evidenciando a necessidade de um aprofundamento das questões surgidas sobre as reações emocionais, tendo nas ações de autocuidado uma abordagem que dê conta de enfrentar as interferências geradas pelos sentimentos envolvidos no contexto dos cuidados de enfermagem aos portadores de DM.

9. Rodrigues WHC. Papéis de autocuidado para o escolar portador de diabetes mellitus tipo 1: uma abordagem de enfermagem 2006. [relatório de qualificação de dissertação de mestrado]. Rio de Janeiro (RJ): Escola de Enfermagem Anna Nery/UFRJ; 2006. p. 48-58.

10. Santos CVF. A consulta de enfermagem e os papéis de autocuidado na prevenção do pé diabético [relatório de qualificação de dissertação de mestrado] Rio de Janeiro (RJ): Escola de Enfermagem Anna Nery/ UFRJ; 2006. p.57-69.

11. Ministério da Saúde (BR). Conselho Nacional de Saúde. Diretrizes e normas regulamentadoras de pesquisas envolvendo seres humanos. Resolução CNS n¹96, de 10 de outubro de 1996. InfEpidemiol SUS 1996; 5 (2 supl 3): 13-41.

12. Chiozza $L A$, organizador. Psicanálise do distúrbio diabético. In Os afetos ocultos em psoríase, asma, transtornos respiratórios, varizes, diabetes, transtornos ósseos, cefaléias e acidentes cerebrovasculares. São Paulo (SP): Casa do Psicólogo; 1997 p. 115-43.

13. Dakle R. A doença como símbolo: pequena enciclopédia de psicossomática, sintomas, significados, tratamentos e remissão. São Paulo(SP): Cultrix, 1996. p.334

14. Laplantine F. Antropologia da doença. São Paulo (SP): Martins Fontes; 1991. p. 274 